\title{
Correlations of Relative Sensitivities in Gas Chromatography Electron Ionization Mass Spectrometry with Molecular Parameters
}

\author{
Charles Allgood*, Ronald Orlando**, and Burnaby Munson \\ Department of Chemistry and Biochemistry, University of Delaware, Newark, Delaware, USA
}

\begin{abstract}
The relative molar sensitivities for a number of compounds having a variety of functional groups were obtained in gas chromatography electron ionization mass spectrometry. Comparable results were obtained with a quadrupole and with a magnetic mass spectrometer. The present relative molar sensitivities are in good agreement with relative ionization cross sections obtained by different techniques and different instruments for a variety of compounds with molecular weights below about $200 \mathrm{u}$. For compounds of higher molecular weight, the present experimental sensitivities are significantly larger than estimates extrapolated from earlier data. The relatively molar sensitivities correlate well with molecular polarizability. ( $J$ Am Soc Mass Spectrom 1990, 1, 397-404)
\end{abstract}

$\mathrm{O}$ ne problem in the quantitation of complex mixtures by any technique is the uncertain availability of standards that are needed to establish the relative sensitivities for the components of the mixtures. The more complex the mixture, the less likely one is to obtain all of the components. Methods of correlating relative sensitivities for compounds with molecular structures are of great importance in reducing the necessary number of standardization experiments. With the extensive use of gas chromatography/electron ionization mass spectrometry (GC/EIMS) for the quantitative analysis of complex mixtures, the ability to predict the sensitivity of a compound on the basis of easily obtainable molecular parameters would be extremely useful, particularly for environmental or fossil fuel samples for which individual components are not available.

It was shown many years ago that the total ionization for isomeric hydrocarbons (relative to $n$-butane) obtained with $50-75-\mathrm{eV}$ electrons is constant within $\pm 5 \%$ for many isomeric alkanes and a few isomeric alkenes and alkylbenzenes [1]. It was also observed that the total ionization per unit sample pressure increased with increasing carbon number in an homologous series [1]. A plot of total ion current vs. carbon number leveled off at about $C_{10}$, although there was

\footnotetext{
*Present address: Organic Analytical Research Division, NIST, Gaithers burg, MD 20899.

** Present address: Department of Chemistry and Biochemistry. University of Maryland, Baltimore County, Baltimore, MD 21228.
}

Address reprint requests to Burnaby Munson, Department of Chemistry and Biochemistry, University of Delaware, Newark, DE 19716. no obvious explanation for this trend [1, 2]. In addition, it was noted that the total ionization decreased with increasing unsaturation for hydrocarbons having the same number of carbon atoms [1, 2].

If there is no significant mass discrimination in the mass spectrometer, then the total sample ion current measured at the detector in the analytical experiments discussed above is proportional to the amount of total sample ionization in the source of the mass spectrometer, with the same proportionality constants for all compounds. Hence the ratio of the total "analytical" ionization of two samples is equal to the ratio of the ionization cross sections of the molecules.

Molecular ionization cross sections with $70 \mathrm{eV}$ electrons have been obtained for many relatively simple molecules at several laboratories [3-7]. These ionization cross sections have been reported for a wide variety of compounds having low to moderate molecular weights-less than $200 \mathrm{u}$. In addition, ionization gauge response factors have been measured for an extensive set of low molecular weight compounds [8]. Recently, GC/EIMS response factors have also been reported for a series of polychlorobiphenyls [9].

Linear correlations have been reported between ionization cross sections and the sum of atomic cross sections $[3,9,10]$, diamagnetic susceptibilities [6], and molecular polarizabilities [4-6]. The best correlations have been obtained between molecular ionization cross sections and polarizabilities, but the ionization cross sections for different compound types do not fit a single correlation with molecular polarizability $[5,6]$. Plots of ionization cross section vs. carbon number for these compounds give good straight lines for different homologous series, with essentially the same in- 
crement per $\mathrm{CH}_{2}$ group [5, 7], in contrast to the nonlinearity with increasing carbon number for the total ionization from the analytical experiments mentioned previously $[1,2]$.

The determinations of ionization cross sections or molar sensitivities by the procedures described above are time consuming. The experimental techniques are not likely to be applied to relatively high molecular weight, low vapor pressure materials. In this article we report data on relative molar sensitivities from simple experiments with a readily available, standard GC/MS system for comparison with the molecular ionization data obtained from the experiments discussed above and to extend the analysis to compounds of higher molecular weight.

\section{Experimental}

Most of the spectral data were acquired with a Hewlett-Packard 5970 Mass Selective Detector (HP MSD, Palo Alto, CA) operated under standard electron ionization conditions. The quadrupole mass filter was tuned using the automatic tuning procedure ( $\mathrm{Au}$ totune) each day of experiments, and a standard spectrum of perfluorotributylamine (PFTBA) was obtained. The measured abundances for the ionic species at $m / z$ 69, 70, 219, 220, 502, and 503 in PFTBA were recorded for each set of tuning conditions.

Sample solutions of accurately known concentrations in ethanol were prepared from commercially available pure compounds, approximately $0.1 \mathrm{M}$. A sample of approximately $1 \mu \mathrm{L}$ of each solution was injected into the gas chromatograph (HP 5980A), which was operated in the split injection mode. These samples were separated on a $12 \mathrm{~m} \times 0.2 \mathrm{~mm} \times 0.33 \mu \mathrm{m}$ film thickness cross-linked methyl silicone capillary column and introduced directly into the source of the mass spectrometer. The temperature of the oven of the gas chromatograph was programmed to achieve complete separation of all components of each mixture.

The total ion current (TIC) was recorded over the complete mass range of the samples $(20-500 \mathrm{u}$ ), and the sensitivity was obtained by integrating the total ion current across the chromatographic peak for each compound. The baseline of each peak was determined by the computer for each individual component of the mixture in order to compensate for any changes in the background because the major air peaks $(\mathrm{m} / z 28$ and 32) were included in every scan. All calculations were done using the standard software package provided with the HP MSD. All values are reported relative to an internal standard: $n$-octane, $n$-decane, or ethylbenzene. The reported values of relative sensitivities are the averages of at least three separate GC/EIMS experiments. Approximately $5 \mathrm{~s}$ was required to obtain a full range mass spectrum $(20-500 \mathrm{u})$ with a time of approximately $0.5 \mathrm{~s}$ between mass spectra. Some 10-12 spectra were obtained across each chromatographic peak un- der these conditions. However, using as few as six or as many as $\mathbf{2 0}$ mass spectra had no significant effects on the average relative peak areas or on the standard deviations of the measurements.

The average molecular polarizabilities were calculated from atomic hybrid components [11]. These values are somewhat different from those calculated from bond polarizability data [12], but the polarizability ratios are essentially identical when calculated by either method. The data were analyzed and plotted by means of a standard curve-fitting routine (F-Curve, LEDS Publ. Co., Research Triangle Park, NC).

In order to assure that the sample sizes routinely being used in these experiments were in the linear working range of the mass spectrometer system, plots were made of total ionization vs. sample mass for a few mixtures. These plots gave good straight lines that yielded the relative sensitivities from the ratios of the slopes. The relative sensitivities obtained in this manner agreed well with those obtained from the total ion current ratios for mixtures of known concentration.

The tuning parameters (Autotune) for the $\mathrm{HP}^{\mathrm{P}}$ MSD varied significantly over the period in which these measurements were made. However, the relative abundances of the fragment ions of the calibration compound, PFTBA, remained relatively constant.

In Table 1 the short-term ( 1 hour), medium-term (8 hours), and long-term (1 week) reproducibility of this technique are shown. The average standard deviation was $\pm 3 \%$ for the short-term and $\pm 15 \%$ for the long-term experiments. The average values are in good agreement and do not differ by amounts that are significantly larger than the standard deviations of the measurements.

In order to assure that no losses were occurring because of the adsorption of high molecular weight compounds in the ion source or the GC/EIMS transfer line, experiments were performed in which the temperature of the ion source and transfer line was varied while all other instrumental parameters were kept con-

Table 1. Reproducibility of relative sensitivities ${ }^{a}$

\begin{tabular}{lccc}
\hline Compound & $\begin{array}{c}\text { Short } \\
\text { term }^{b}\end{array}$ & $\begin{array}{c}\text { Medium } \\
\text { term }^{c}\end{array}$ & $\begin{array}{c}\text { Long } \\
\text { term }\end{array}$ \\
\hline \hline N,N-dimethylaniline & $0.98(.03)$ & $1.02(.05)$ & $1.04(.07)$ \\
$n$-Tridecane & $1.40(.07)$ & $1.41(.07)$ & $1.20(.15)$ \\
Ethyl formate & $0.20(.01)$ & $0.19(.02)$ & $0.17(.04)$ \\
c-Hexene & $0.53(.01)$ & $0.49(.05)$ & $0.45(.09)$ \\
Methyl benzoate & $1.09(.02)$ & $1.20(.09)$ & $1.20(.09)$ \\
\hline
\end{tabular}

${ }^{\circ} \mathrm{GC} /$ EIMS un an HP MSD. All values relative tu $n$-decane $=1.00$. Standard deviations given in parentheses.

${ }^{b}$ Average of five replicate GC / MS analyses in a one-hour period using the same instrumental tuning parameters.

Average of five replicate GC/MS analyses over an eight-hour period using the same instrumental tuning parameters.

Average of four sets of five replicate GC / MS analyses over a one-week period with different optimum tuning parameters for each set of experiments. 
Tabie 2. Effect of GC/MS temperatures on relative sensitivities for some phthalate esters

\begin{tabular}{lcccc}
\multicolumn{2}{c}{ Temperatures } & \multicolumn{3}{c}{ Phthalate esters $^{c}$} \\
\cline { 3 - 5 } $\mathrm{TL}^{\mathrm{a}}$ & $\mathrm{IP}^{\mathrm{b}}$ & Diethyl & $n$-Butyl & $n$-Amyl \\
\hline \hline 240 & 250 & $1.55(.17)$ & $2.11(.21)$ & $2.30(.23)$ \\
280 & 250 & $1.60(.16)$ & $2.20(.28)$ & $2.40(.11)$ \\
280 & 250 & $1.76(.14)$ & $2.05(.11)$ & $2.43(.13)$ \\
280 & 250 & $1.72(.08)$ & $2.22(.23)$ & $2.43(.25)$ \\
320 & 250 & $1.72(.26)$ & $2.28(.29)$ & $2.56(.25)$
\end{tabular}

"TL = Transfer line temperature, ${ }^{\circ} \mathrm{C}$, for HP MSD.

${ }^{\circ} \mathrm{IP}=$ Injection port temperature, ${ }^{\circ} \mathrm{C}$, for HP MSD.

${ }^{\mathrm{c}}$ Sensitivity relative to ethylbenzene. Average of three values. Standard deviations given in parentheses.

stant. The results for a mixture of several aromatic esters are shown in Table 2. Here ethylbenzene was used as an internal standard. The mixture was analyzed at temperatures $40^{\circ} \mathrm{C}$ lower and $40^{\circ} \mathrm{C}$ higher than the standard operating temperature $\left(280^{\circ} \mathrm{C}\right)$. The values for the relative sensitivities for the higher molecular weight, less volatile esters were somewhat lower in the experiments having the lowest temperature $\left(240^{\circ} \mathrm{C}\right)$ than at the higher temperatures. However, there were no majur variations within this range of temperature, and there are no indications of significant adsorption losses for these compounds at the temperature routinely used in these experiments $\left(280^{\circ} \mathrm{C}\right)$.

In the normal operation of the HP MSD, the current through the filament was turned off for a few minutes while the large quantities of solvent vapor pass through the ionization chamber. The duration of this passage is termed the solvent delay time. Several GC/EIMS experiments were performed with various solvent delay times. Other experiments were performed with neat mixtures of compounds involving no solvent delay times. The relative molar sensitivities were not dependent upon these experimental parameters.

Other mass spectrometric experiments were performed with a Du Pont 492B mass spectrometer (Wilmington, DE) interfaced without a separator to a Varian Moduline $2740 \mathrm{GC}$ system (Palo Alto, CA) having a $3 \%$ SP-2100 packed column and helium as the carrier gas. Neat mixtures with injection volumes of $0.05 \mu \mathrm{L}$ were used with this instrument. The data were collected with an IBM AT computer interfaced to the mass spectrometer by a Teknivent data system (Teknivent, Inc., St. Louis, MO). This instrument has been shown to be free of mass discrimination in the mass range of these experiments [13]. Approximately eight fullrange mass spectra $(20-800 \mathrm{u})$ were collected for each chromatographic peak. Additional samples were introduced directly into the source of this instrument from a glass capillary in a Vespel probe.

A comparison for one set of compounds obtained with two very different GC/EIMS systems is presented
Table 3. Experimental sensitivities obtained with two different GC/MS systems ${ }^{a}$

\begin{tabular}{lcc}
\hline Compound & HP MSD & Du Pont 492 \\
\hline \hline$n$-Octane & $0.71(.06)$ & $0.80(.05)$ \\
$n$-Nonane & $0.82(.09)$ & $0.85(.07)$ \\
$n$-Undecane & $1.23(.22)$ & $1.14(.08)$ \\
$n$-Dodecane & $1.37(.16)$ & $1.31(.08)$ \\
Chlorobenzene & $0.59(.04)$ & $0.43(.05)$ \\
$n$-Propylbenzene & $0.63(.09)$ & $0.77(.05)$ \\
5-Nonanone & $0.70(.11)$ & $0.81(.16)$ \\
\hline
\end{tabular}

${ }^{a}$ Average of five experiments obtained during one dav. The standard deviations are given in parentheses. Values are reported relative to $n$-decane $=1$. 00 .

bHewlett-Packard Mases Selective Detector (quadrupole mass spectrometer) with capillary gas chromatographic column.

${ }^{\circ}$ Du Pont $492 \mathrm{~B}$ double-focusing magnetic mass spectrometer with packed column without a separator.

in Table 3: a capillary GC column with a quadrupole mass spectrometer and a packed GC column with a magnetic mass spectrometer. The differences in the individual relative sensitivities were less than the combined relative standard deviations of the experiments, except for chlorobenzene. The average value of the ratios of the relative sensitivities was very close to unity, $1.02 \pm 0.07$. This good agreement between the data obtained with these two very different GC/EIMS systems indicates that there were no significant discrimination effects or differential loss processes in these experiments.

An additional set of experiments was performed to validate the relative sensitivities obtained by GC/EIMS with the HP MSD. An accurately known mixture of 1-methylphenanthrene, 9,10-benzopheranthrene, and perylene was completely evaporated from a glass capillary in a heated probe using the Du Pont 492B mass spectrometer by raising the temperature of the probe over a period of several minutes. The total ion currents for the $\mathrm{M}^{+}$and the few characteristic fragment ions were obtained for each compound. The ratio of total ion currents (corrected to the same number of moles) is the ratio of molar sensitivities. Relative molar sensitivities were also obtained for these mixtures in GC/EIMS experiments with the HP MSD.

The molar sensitivity for 1-methylphenanthrene relative to perylene was determined to be $0.72 \pm 0.12$ from the probe distillation experiments with the magnetic mass spectrometer and $0.71 \pm 0.14$ from the GC/EIMS experiments with the quadrupole mass spectrometer. The molar sensitivity for 9,10benzophenanthrene relative to perylene was determined to be $0.76 \pm 0.15$ from the GC/EIMS experiments and $0.84 \pm 0.05$ from the probe distillation experiments.

These experiments established the absence of significant mass discrimination or differential loss processes in the determination of relative abundances in 
the GC/EIMS experiments using the HP MSD and capillary gas chromatograph. Consequently, the data reported subsequently were all obtained with this system.

\section{Results and Discussion}

As indicated previously, at the low pressures of EI experiments the total ion current is directly related to the ionization cross section $[3-7,9]$, by the equation

$$
\Sigma I_{i}^{+}=Q_{i} I_{e}\left[M_{i}\right] d
$$

in which $\Sigma I_{i}^{+}$is the total positive ion current, $Q_{i}$ is the ionization cross section, $\left[M_{i}\right]$ is the concentration of sample molecules of species $i, I_{e}$ is the electron cur rent, and $d$ is the ionization path length. With a constant electron current and path length the ionization cross sections can be determined from the slopes of plots of total sample ionization vs. concentration as indicated by eq 1 . In the earlier work the absolute values of ionization cross sections were calculated by using the absolute value of the ionization cross section for argon or krypton from other experiments [3-7].

In the present GC/EIMS experiments the total ion current (TIC) was obtained for each mass spectrum by summing the ion currents of all sample ions registered at the detector. If there are no mass discrimination effects in the mass spectrometer, this TIC measured at the detector is proportional to the TIC in the source, with the same proportionality constant for all masses:

$$
\mathbf{T I C}=a \Sigma I_{i}^{+}
$$

The concentration of the sample is a timedependent quantity across a chromatographic peak, related to the total number of moles of material, $N_{i}$, and the volume of the ionization chamber, $V$ :

$$
N_{i}=\int\left[M_{i}\right] V d t=\int\left(P_{i} V / R T\right) d t
$$

The TICs for each mass spectrum for a component $i$ obtained during the elution of the compound from the chromatograph were summed to give a total area, $A_{i}$, which is directly proportional to the amount of sample and to the molar sensitivity $\left(S_{i}\right)$ or to the ionization cross section:

$$
A_{i}=S_{i} N_{i}=k Q_{i} N_{i}\left(I_{e} d / a V\right)
$$

From the ratios of total areas for mixtures of known composition, $N_{i}$ and $N_{j}$, we calculated the ratios of molar sensitivities, $S_{i} / S_{j}$, or of ionization cross sections, $Q_{i} / Q_{j}$ :

$$
S_{i} / S_{j}=\left(A_{i} / N_{i}\right) /\left(A_{j} / N_{j}\right)=Q_{i} / Q_{j}
$$

In Table 4 the relative molar sensitivities from the present experiments are compared with previously reported ionization cross sections for several compounds. The ionization cross sections of Harrison et al. [5] and Alberti et al. [7] were obtained by the method discussed above. The data of Bartmess and Georgiadis [8] are ionization gauge sensitivities, which correlate

\begin{tabular}{|c|c|c|c|c|c|}
\hline Compound & This work & Harrison et al. ${ }^{a}$ & Bartmess and Georgiadis $^{b}$ & Mohler et al. ${ }^{\mathrm{c}}$ & Alberti et al. ${ }^{d}$ \\
\hline n Octane & $1.27(.06)$ & 1.31 & - & 1.59 & 1.31 \\
\hline$n$-Nonane & $1.49(.13)$ & 1.42 & - & 1.72 & 1.47 \\
\hline$n$-Decane & $1.63(.11)$ & 1.58 & - & 1.56 & 1.62 \\
\hline n-Undecane & $1.86(.10)$ & - & - & 1.72 & 1.77 \\
\hline r-Dodecane & $2.16(.10)$ & - & - & 1.69 & 1.93 \\
\hline o-Xylene & $0.99(.03)$ & 0.98 & 0.91 & - & - \\
\hline Ethylbenzene & 1.00 & 1.00 & 1.00 & 1.00 & 1.00 \\
\hline Propylbenzene & $1.28(.09)$ & 1.17 & - & 1.10 & 1.15 \\
\hline n-Butylbenzene & $1.42(.08)$ & 1.27 & - & - & 1.30 \\
\hline$t$-Butylbenzene & $1.48(.15)$ & 1.27 & - & - & - \\
\hline Anisole & $0.89(.03)$ & - & 0.72 & - & - \\
\hline Ethyl formate & $0.24(.04)$ & 0.57 & 0.46 & - & - \\
\hline Ethyl acetate & $0.51(.10)$ & 0.75 & 0.54 & - & - \\
\hline$c$-Hexene & $0.67(.08)$ & 0.82 & 0.80 & - & - \\
\hline
\end{tabular}
well with ionization cross sections. The data of Mohler et al. [1] are relative total ionizations obtained from samples introduced through a heated oven. All val-

Table 4. Comparison of relative sensitivies

Data taken from ref 5

bata taken from ref $\mathbf{8}$.

"Data taken from ref 1 . Sensitivity for ethylbenzene taken as the average of values for $C_{B}$-alkylbenzenes.

dData taken from ref 7 . 
ues have been calculated relative to ethylbenzene as a common standard. Care must be taken in direct comparisons of ionization cross sections because different values were used for the reference compounds.

The average relative standard deviation for the data from the present GC/EIMS experiments listed in Table 4 is approximately $\pm 5 \%$; however, a more realistic estimate is $\pm 15 \%$, as indicated by the long-term precision of the data in Table 1. The estimates of the short-term precision of the measurements by the other workers are $\pm 3-5 \%$.

As noted earlier, the data of Mohler et al. [1] for the alkanes do not show the increase with increasing molecular weight that is observed in the data from the other three laboratories. It is very possible that there were sample introduction problems for the higher molecular weight compounds in the earlier studies that gave these low results. The agreement of the present data with the other three groups is good. The present data appear to be slightly higher than the data of Alberti et al. [7] for the highest molecular weight alkanes and alkylbenzenes. However, the differences are marginally larger than the combined uncertainties of the measurements. The present value for the relative sensitivity for ethyl formate is significantly lower than the literature values and may be in error.

These results indicate that reliable values for relative molecular ionization cross sections can be measured with a standard quadrupole mass spectrometer without the specific instrumental corrections reported previously [9]. An extensive set of relative molar sensitivities (relative ionization cross sections) was obtained, mostly for hydrocarbons and oxygenated compounds, including a few homologous series. These values, all relative to $n$-octane $=1.00$, are given in Table 5 .

Also included in Table 5 and plotted in Figure 1 are the relative sensitivities per gram for these compounds. A small increase in the relative sensitivity per gram with increasing molecular weight in the 200-500 $u$ range is indicated by the solid line, which shows a least squares analysis of the data. However, within the precision of the data one can also postulate that the relative sensitivity per gram is constant and independent of molecular structure and molecular weight. The average of the relative sensitivities per gram for all of the 58 compounds in Table 5 is $0.96 \pm 0.16$. Although the great majority of these compounds were hydrocarbons and oxygenated derivatives, the constancy of the relative sensitivity per gram appears to hold for the few sulfur compounds and the nitrogen compound in Table 5.

Among the present compounds the lower molecular weight oxygenated compounds have relative sensitivities per gram that are consistently lower than the average by amounts that are somewhat greater than the standard deviation. The two bromine-containing compounds are also clear exceptions to the general trend. The relative sensitivities per gram for the compounds
Table 5. Relative sensitivities

\begin{tabular}{|c|c|c|c|c|}
\hline \multirow[b]{2}{*}{ Compound } & \multirow[b]{2}{*}{$P^{\mathrm{b}}$} & \multirow[b]{2}{*}{ MW } & \multicolumn{2}{|c|}{ Relative sensitivity } \\
\hline & & & Molar & Gram \\
\hline n-Octane & 1.000 & 114.2 & 1.00 & 1.00 \\
\hline n-Nonane & 1.120 & 128.2 & $1.18(.10)$ & 1.05 \\
\hline$n$-Decane & 1.240 & 142.3 & $1.29(.09)$ & 1.03 \\
\hline$n$-Undecane & 1.360 & 156.3 & $1.47(.07)$ & 1.07 \\
\hline n-Dodecane & 1.480 & 170.3 & $1.71(.09)$ & 1.15 \\
\hline$n$-Tridecane & 1.600 & 184.3 & $1.95(.07)$ & 1.20 \\
\hline n-Hexadecane & 1.957 & 226.4 & $2.10(.19)$ & 1.06 \\
\hline n-Octadecane & 2.196 & 254.5 & $2.79(.31)$ & 1.25 \\
\hline Ethylbenzene & 0.870 & 106.2 & $0.79(.05)$ & 0.84 \\
\hline$m$-Xylene & 0.870 & 106.2 & $0.80(.05)$ & 0.85 \\
\hline$o$-Xylene & 0.870 & 106.2 & $0.78(.02)$ & 0.85 \\
\hline n-Propylbenzene & 0.990 & 120.2 & $1.01(.07)$ & 0.96 \\
\hline n-Butylbenzene & 1.110 & 134.2 & $1.12(.06)$ & 0.96 \\
\hline$t$-Butylbenzene & 1.110 & 134.2 & $1.17(.12)$ & 1.00 \\
\hline 5-t-Butyl $m$-xylene & 1.350 & 162.3 & $1.44(.15)$ & 1.00 \\
\hline Di-i-propylbenzene & 1.350 & 162.3 & $1.62(.20)$ & 1.14 \\
\hline n-Hexylbenzene & 1.350 & 162.3 & $1.58(.16)$ & 1.10 \\
\hline n-Decylbenzene & 1.832 & 218.4 & $2.20(.26)$ & 1.15 \\
\hline c-Pentanone & 0.590 & 84.1 & $0.53(.04)$ & 0.72 \\
\hline$c$-Hexanone & 0.740 & 98.1 & $0.68(.09)$ & 0.79 \\
\hline 5-Nonanone & 1.124 & 142.2 & $0.97(.13)$ & 0.78 \\
\hline 2-Undecanone & 1.360 & 170.3 & $1.53(.16)$ & 1.03 \\
\hline$o$-Cresol & 0.760 & 110.1 & $0.66(.09)$ & 0.70 \\
\hline$p$-Cresol & 0.760 & 110.1 & $0.71(.13)$ & 0.75 \\
\hline Anisole & 0.804 & 108.1 & $0.70(.02)$ & 0.74 \\
\hline Acetophenone & 0.881 & 120.2 & $0.79(.08)$ & 0.75 \\
\hline Butyrophenone & 1.118 & 148.2 & $1.04(.04)$ & 0.80 \\
\hline i-Valerophenone & 1.237 & 162.2 & $1.14(.13)$ & 0.80 \\
\hline Hexanophenone & 1.356 & 176.3 & $1.42(.09)$ & 0.92 \\
\hline Heptanophenone & 1.476 & 190.3 & $1.62(.11)$ & 0.97 \\
\hline Octanophenone & 1.595 & 204.3 & $1.92(.10)$ & 1.07 \\
\hline Nonanophenone & 1.714 & 218.3 & $2.10(.14)$ & 1.09 \\
\hline Chlorobenzene & 0.800 & 112.6 & $0.77(.06)$ & 0.77 \\
\hline Bromobenzene & 0.856 & 157.0 & $0.81(.12)$ & 0.58 \\
\hline$o$-Dibromobenzene & 1.054 & 235.9 & $1.08(.05)$ & 0.52 \\
\hline i-Propylsulfide & 0.961 & 118.2 & $0.92(.01)$ & 0.90 \\
\hline Hexanethiol & 0.961 & 118.2 & $0.91(.01)$ & 0.88 \\
\hline Octanethiol & 1.200 & 146.3 & $1.25(.04)$ & 0.98 \\
\hline $\mathrm{N}, \mathrm{N}$-dimethylaniline & 1.001 & 121.2 & $1.09(.08)$ & 1.03 \\
\hline i-Propyl benzoate & 1.164 & 164.2 & $1.41(.03)$ & 0.98 \\
\hline Diethyl phthalate & 1.453 & 222.2 & $1.60(.16)$ & 0.82 \\
\hline Di $n$ butyl phthalate & 1.927 & 278.3 & $2.23(.28)$ & 0.91 \\
\hline Di-amyl phthalate & 2.165 & 306.4 & $2.46(.14)$ & 0.91 \\
\hline
\end{tabular}

Ethoxylated alcohols

$$
\begin{array}{rrrrr}
\hline \mathrm{C}_{n} \mathrm{H}_{2 n+1} \mathrm{O}_{\left(\mathrm{CH}_{2} \mathrm{CH}_{2} \mathrm{O}\right)} \mathrm{H} & & & \\
n=10 \quad x=1 & 1.565 & 202.3 & 1.63(.18) & 0.92 \\
x=2 & 1.848 & 246.4 & 2.23(.52) & 1.03 \\
x=3 & 2.132 & 290.4 & 3.13(.60) & 1.23 \\
& & & & \\
\text { (Continued) }
\end{array}
$$


Table 5. (Continued)

\begin{tabular}{rllll}
\hline & & \multicolumn{3}{c}{ Relative sensitivity } \\
\cline { 3 - 6 } Compound & $P^{\mathrm{b}}$ & $\mathrm{MW}$ & Molar & Gram \\
\hline \multicolumn{1}{c}{$x=6$} & 2.982 & 422.6 & $4.07(.24)$ & 1.10 \\
$x=8$ & 3.549 & 510.7 & $4.25(.42)$ & 0.95 \\
$n=12 \quad x=1$ & 1.801 & 230.4 & $2.10(.29)$ & 1.04 \\
$x=3$ & 2.368 & 318.5 & $2.69(.45)$ & 0.96 \\
$x=4$ & 2.652 & 362.5 & $3.44(.42)$ & 1.08 \\
$x=5$ & 2.935 & 406.6 & $3.95(.55)$ & 1.11 \\
$x=6$ & 3.218 & 450.6 & $4.54(.47)$ & 1.15 \\
$n=14 x=1$ & 2.038 & 258.4 & $2.16(.22)$ & 0.91 \\
$x=2$ & 2.321 & 302.5 & $2.96(.46)$ & 1.12 \\
$x=3$ & 2.605 & 346.5 & $3.56(.51)$ & 1.17 \\
$x=6$ & 3.455 & 478.7 & $4.43(.55)$ & 1.06 \\
\hline
\end{tabular}

${ }^{2}$ Average of at least three GC/MS experiments. Standard devia tions given in parentheses.

Dolarizability ratio, calculated according to ref 11 .

in this study show a much smaller variation than that reported recently for response factors in other GC/EIMS work for a set of chlorinated biphenyls [9]. We also note that values of $Q_{i} / M_{i}$ (which are proportional to the relative sensitivities per gram) for several low molecular weight perfluorocarbons, fluorochlorocarbons, and fluorobromocarbons are significantly lower than the average value for $Q_{1} / \mathrm{MW}_{1}$ for hydrocarbons [6]. Values of $\left[Q_{i} / \mathrm{MW}_{i}\right] /[Q(n$-octane $) / \mathrm{MW}(n-$ octane)] for sets of alkyl fluorides, chlorides, bromides, and iodides are significantly less than one and increase with increasing number of methylene groups [7]. Halogenated organic compounds do not fit this pattern of a constant relative sensitivity per gram.

These data on EI relative sensitivity per gram can be compared with weight response factors (sensitivities per gram) obtained with a flame ionization detector (FID). The relative FID sensitivities per gram for hydrocarbons are essentially constant and independent of molecular weight and hydrocarbon type, as is observed with the present EI relative sensitivities per gram; however, there is a much wider range in FID relative sensitivities per gram for low molecular weight oxygen- and nitrogen-containing compounds-as large as a factor of three $[14,15]$. Within an homologous series of compounds containing a single functional group, the FID relative sensitivities per gram increase with increasing carbon number and approach (but are always less than) the value for a hydrocarbon having the same number of carbon atoms [16]. From the effective carbon number rule one estimates, for example, that ethers and ketones have FID relative sensitivities per gram of $(a-1) / a$, where $a$ is the number of carbon atoms in the molecule $[15,16]$. Consequently, for compounds containing more than $\mathbf{1 0}$ or so carbon atoms the FID relative sensitivities per gram will be insensitive to molecular weight, as noted for the EIMS sensitivities per gram. For a series of oligomeric ethoxy- lated alcohols, however, the FID relative sensitivities per gram can be correlated with the ratio of the number of oxygen to carbon atoms, but they vary quite widely-by approximately a factor of two for $C_{10}$ to $C_{16}$ alcohols having 1-8 ethylene oxide units [17]. This variation is much larger than the variation in EIMS relative sensitivities per gram for similar compounds indicated in Table 5.

We note, in agreement with earlier work on ionization cross sections $[1,5]$, that the relative molar sensitivities for the skeletal and positional isomers of the alkylbenzenes do not differ by amounts larger than the standard deviations of the measurements. Similarly, the relative molar sensitivities for the few other isomeric compounds in these experiments do not differ by amounts larger than the precision of the measurements. The indistinguishability of the relative molar sensitivities of the functional isomers, hexanethiol and di-i-propyl sulfide, may be different from the earlier reports of different ionization cross sections for functional isomers of oxygenated compounds [5]. However, we note that the previously reported small differences $(1-8 \%)$ were only slightly larger than the uncertainties of the measurements and are smaller than the uncertainties in the present data.

Correlations have been reported previously between iorization cross sections and molecular polarizabilities, although it was noted that no single correlation fitted the data for all classes of compounds [4-6]. The correlations have generally been linear, although not always giving a good fit and a zero intercept for each class of compounds. Figure 2 shows a plot of the relative molar sensitivities against the polarizability ratios for these data. The linear correlation is good $(R=0.991)$, with an average difference between the calculated and experimental data points of $5.3 \%$. However, the slope of this curve is not unity (1.37), and the intercept is not zero $(-0.440)$. The plot of the data in Figure 1 appears to have some curvature and can be fitted reasonably well by the equation

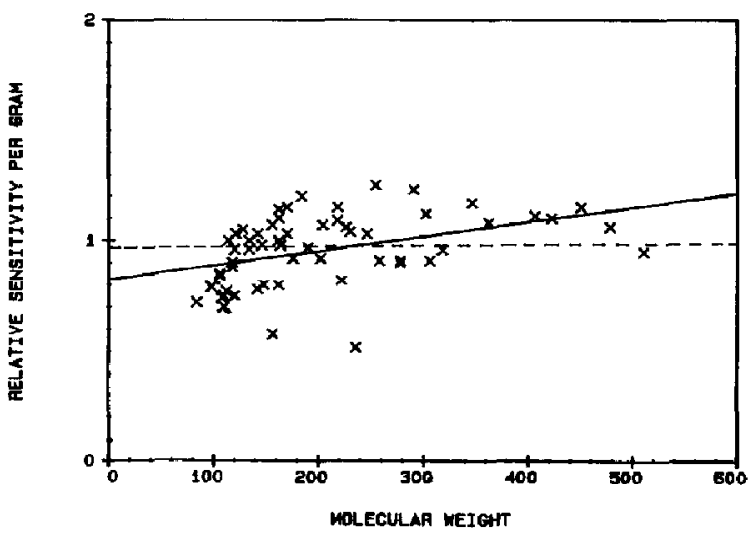

Figure 1. Relative sensitivity per gram vs. molecular weight. All values relative to $n$-octane $=1.000$. Solid line is a least squares fit to data. Dashed line is a constant relative sensitivity per gram. 


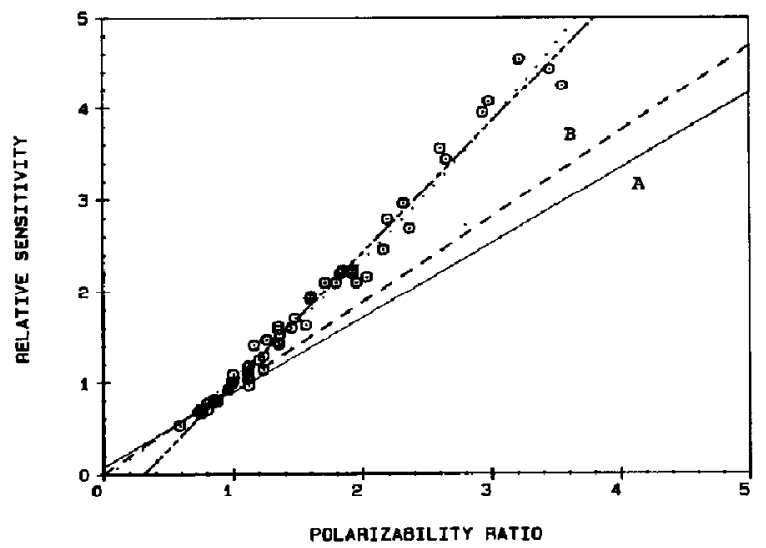

Figure 2. Relative molar sensitivity vs. polarizability ratio. All values relative to $n$-octane $=1.000$. Line $A$ from ref 5 . Line $B$ from ref 7 .

$Y=a X+b X^{2}$ (dotted line; $a=0.836, b=0.163$, $Y=$ relative sensitivity, $X=$ polarizability ratio). The calculated values of relative molar sensitivities differ from the experimental values by an average of $6.2 \%$. The few halogenated compounds in this study fitted the correlation of relative molar sensitivity against polarizability ratio as well as the other compounds.

Also shown in Figure 2 are plots of previous data, which could be directly normalized to $n$-octane for comparison with the present data. The relative ionization cross section data of Alberti et al. [7] for RX compounds ( $\left.\mathrm{X}=\mathrm{CH}_{3}, \mathrm{C}_{6} \mathrm{H}_{5}, \mathrm{~F}, \mathrm{Cl}, \mathrm{Br}, \mathrm{I}\right)$ give a good correlation with polarizability ratio as indicated by the dashed line $(R=0.987$, slope $=0.935$, intercept $=$ $0.010,6.8 \%$ relative standard deviation of points from the straight line), although the data can be fitted better by a set of lines for each functional group. The data of Harrison et al. [5] for a wider series of functional groups, mostly hydrocarbons and oxygenated hydrocarbons, also give a good correlation with polarizability ratio as indicated by the solid line $(R=0.970$, slope $=0.816$, intercept $=0.083,7.3 \%$ relative standard deviation of points from the straight line). Again, the data can be fitted better by a set of lines for different functional groups. Use of normalized data eliminates the complications resulting from differences in choices of reference compounds for the absolute ionization cross sections. The data of Alberti et al. [7] and of Harrison et al. [5] overlap in the region of polarizability ratios of 0.2 to 1.2 , and although the curves are distinguishable, the data do not appear to differ by amounts that exceed the combined uncertainties of the measurements. Our data overlap those of Harrison in the region of polarizability ratios of 0.6 to 1.2 and are not different by amounts that are larger than the combined uncertainties in the data. Our data overlap those of Alberti in the region of polarizability ratios of 0.6 to 1.8 , and only at the upper end of this range is there any significant difference between the two sets of data. The present data, however, are clearly different from the extrapolations of the previous data to higher molecular weights (larger polarizability ratios).

In agreement with previous observations on ionization cross sections $[5,7]$, we note that the relative molar sensitivities increase linearly with increasing carbon number for compounds of the same type, with essentially the same slope for each class of compound. Recalculating the previous ionization cross section data relative to octane gives an increase of $0.10-0.12$ in relative molecular ionization cross section per methylene group. The present data give larger slopes, 0.15-0.19, for relative molar sensitivities for similar compounds at somewhat higher molecular weights.

A general correlation between carbon number and relative sensitivity exists for the set of all the compounds in this article, but the correlation is not as good as those between relative molar sensitivity and polarizability, or relative molar sensitivity and carbon number, for each class of compounds, evidently because of the neglect of the contributions from the heteroatoms. A reasonably linear correlation exists between relative molar sensitivity and molecular weight; however, the scatter of the data about the line is worse for this correlation than for the correlation with molar polarizability. The halogenated compounds do not fit the correlation with molecular weight: the calculated values are much too high.

\section{Conclusion}

Although individual sets of data, with presumably high accuracy, require multiple correlations for accurate description, the relative molar sensitivities for a large number of organic compounds over a wide molecular weight range can be correlated with reasonable accuracy $(<10 \%)$ with a single molecular property-the polarizability ratio. The relative sensitivities per gram for many hydrocarbons and oxygenated compounds, but not halogenated compounds, are the same.

\section{Acknowledgment}

This work was supported by grant no. CHE 8813619 from the National Science Foundation.

\section{References}

1. Mohler, F.; Williamson, L.; Dean, H. J. Res. Nat. Bur. Stds. 1950, 47, 235.

2. Field, F. H.; Franklin, J. L. Electron Impact Phenomena; Academic: New York, 1957.

3. Otvos, J.; Stevenson, D. P. J. Am. Chem. Soc. 1957, 79, 546.

4. Lampe, F. W.; Franklin, J. L.; Field, F, H. J. Am. Chem. Soc. $1957,79,6129$.

5. Harrison, A.; Jones, E.; Gupta, S.; Nagy, G. Can. J. Chem. 1966, 44, 1967.

6. Kevan, L.; Beran, J. J. Phys. Chem. 1969, 73, 3866.

7. Alberti, R.; Genoni, M. M.; Pascual, C.; Vogt, J. Int. J. Mass Spectrom. Ion Processes 1974, 14, 89.

8. Bartmess, J. E.; Georgiadis, R. M. Vacuum 1983, 33, 149. 
9. Sauter, A. D.; Downs, J. J.; Buchner, J. D.; Ringo, N. T.; Shaw, D. L.; Dulak, J. G. Artal. Chem. 1986, 58, 1665.

10. Fitch, W. L.; Sauter, A. D. Anal. Chem. 1983, 55, 832.

11. Miller, K. J.; Savchik, J. A. J. Am. Chem. Soc. 1979, 101, 7206.

12. Denbigh, K. G. Trans. Far. Soc. 1940, 36, 936.

13. Orlando, R. C.; Munson, B. Anal. Chem. 1988, $60,1815$.

14. Dietz, W. A. J. Gas Chromatography 1967, 5, 68 .
15. Perkins, G. Jr.; Rouayheb, B. M.; Lively, L. D.; Hamilton, W. C. In Gas Chromatography; Brenner, N., Callen، J. E., and Weiss, M. D., Eds.; Academic: New York, 1962; p 269.

16. Sternberg, J. C.; Gallaway, W. S.; Jones, D. T. L. In Gas Chromatography; Brenner, N., Callen, J. E., and Weiss, M. D., Eds.; Academic: New York, 1962; p 231.

17. Geissler, P. R. J. Am. Oil Chern. Soc. 1989, 66, 685. 